\title{
Single-file diffusion in a box: Effect of the initial configuration
}

\author{
S. J. Manzi, J. J. Torrez Herrera, and V. D. Pereyra \\ Departamento de Física, Instituto de Física Aplicada (INFAP)-CONICET, Universidad Nacional de San Luis, \\ Chacabuco 917, 5700 San Luis, Argentina
}

(Received 19 June 2012; published 23 August 2012)

\begin{abstract}
This paper studies the single-file diffusion process on a linear chain of identical pointlike particles with multiple-site occupation confined in a one-dimensional box. The particles are noninteracting, except that double occupancy is forbidden. When particles are confined in a finite box, the final stage is saturation. By means of combinatorial analysis, an exact numerical evaluation of the saturation values for both the mean-square displacement (MSD) of a tracer particle and the center of mass of the system are obtained. Different initial distributions of particles are introduced. The time dependence of the MSD is obtained by means of Monte Carlo simulations. The values of the MSD for the tracer particles as well as the center of mass of the system depend on the size of the particle, the size of the box, and the initial distribution. Moreover, the transient regime depends on the initial distribution. In fact, the crossover from normal to subdiffusive regime is observed for random and alternate initial distributions, while superdiffusive diffusion appears for any stacked initial distributions. In all cases, it is shown that the collisions between particles do not determine the time exponent of the MSD. A simple expression for the transient regime is also obtained for the especial case of random initial distribution.
\end{abstract}

DOI: 10.1103/PhysRevE.86.021129

PACS number(s): 05.50.+q, 68.43.De, 68.43.Jk

\section{INTRODUCTION}

Single-file diffusion (SFD) is a very referenced problem in statistical physics [1-21]. The first report about this phenomena was informed by Hodgkin and Keynes [1], who were studying water transport through molecular-sized channels in biological membranes. New experimental evidence reported in recent years $[2,3,5-8]$ has encouraged the development of new theoretical and numerical studies. It is well known that SFD appears when Brownian particles are confined along a line in a quasi-one-dimensional channel so narrow that they cannot cross each other. As a consequence, anomalous diffusion appears and a strong subdiffusive behavior can be observed. An analytical description of one-dimensional (1D) diffusion in a system of hard rods with stochastic background forces was first reported by Harris [9]. Several other 1D systems have been examined with different approaches [10-14,17,19-24].

The results show that the diffusion of a tagged particle presents a normal diffusion regime [i.e., the mean-square displacement (MSD) increases linearly with time]. This relation is valid up to a characteristic time, beyond which, the dynamic of the system is dominated by particle collisions, leading to single-file behavior (i.e, the MSD is proportional to the square root of time, $\sqrt{t}$ ). This regime depends on the applied boundary conditions of the system, as discussed in Ref. [13]. It is assumed that the subdiffusive regime of a single tagged particle is the signature of the interacting system. On the contrary, the diffusion of the center of mass is always normal, the corresponding MSD increases linearly with time, without showing any signature of the interacting system.

However, this behavior has been observed for random initial distributions of particles. Therefore, it is expected that different initial distributions of particles should have an important influence on the time dependence of the MSD of both the tracer as well as the center of mass of the system. For example, from an initial configuration where the particles are completely stacked, the particles will be forced to move, on average, in one special direction, like in biased diffusion. Then, the time exponent of the MSD should be greater than $1\left[S_{T}(t) \propto t^{\alpha}\right.$ with $\alpha>1]$. Moreover, this superdiffusive behavior should also be observed for the center of mass of the system. However, to our knowledge a detailed study of the influence of the initial distribution of particles in the SFD problem has not been performed yet, in particular, the characterization of the asymptotic regime and time dependence of the MSD for different initial conditions.

Different variants of the SFD problem have been proposed in the literature, for example, the binary mixture problem $[18,20]$ and long-range interactions [25]. The dependence of the SFD on the boundary conditions (confinement) has been analyzed by Lucena et al. [26].

On the other hand, experimental evidence shows diffusion of large molecules in narrow channels, like DNA binding proteins diffusing along a DNA chain [4-6]. From a theoretical point of view, few articles have treated the diffusion of particles with multiple-site occupancy in a one-dimensional chain. In particular, in Ref. [27], the authors have developed a theoretical treatment of the 1D diffusion of $k$-mers (molecules occupying $k$ sites in the chain). However, they have not focalized in the time regime but in the coverage dependence of the diffusion coefficients.

The exact solution for the single-file diffusion process with particles of any size and/or arbitrary initial distribution is rather difficult to obtain, particularly for particles confined in a box. This encourages one to present approximations or numerical simulations to attain a better understanding of the problem, particularly to elucidate the time dependence of the MSD.

This article studies, by means of Monte Carlo simulations, the single-file diffusion process on a linear chain of identical pointlike particles with multiple-site occupation, confined in a finite box. The particles are noninteracting, except that double occupancy is forbidden. Different initial distributions of particles are introduced to analyze the influence on the time behavior of the MSD. By means of combinatorial analysis, an exact numerical evaluation of the saturation values for both the 
MSD of a tracer particle and the center of mass of the system are obtained. The paper is organized as follows: in Sec. II, the basic definitions and the analytical scheme are introduced. In Sec. III, the results of the analytical calculations are presented for different initial distributions and compared with Monte Carlo simulations. Finally, in Sec. IV, the conclusions are presented.

\section{BASIC DEFINITIONS AND ANALYTICAL CALCULATIONS}

The MSD of the center of mass of the system is defined as

$$
S_{J}(t)=\left\langle\left(\sum_{i=1}^{N}\left|\mathbf{r}_{i}(t)-\mathbf{r}_{i}(0)\right|\right)^{2}\right\rangle \text {. }
$$

The above sum can be expressed as

$$
S_{J}(t)=S_{T}(t)+F_{C}(t),
$$

where $S_{T}(t)$ is the MSD of a tagged particles, which is given by

$$
S_{T}(t)=\frac{1}{N} \sum_{i=1}^{N}\left\langle\left[\mathbf{r}_{i}(t)-\mathbf{r}_{i}(0)\right]^{2}\right\rangle,
$$

where $\mathbf{r}_{i}(t)$ is the position of the particle $i$ at time $t$ and $\langle\cdots\rangle$ represents an ensemble average. Expanding the sum in Eq. (3) we obtain

$$
S_{T}(t)=\frac{1}{N}\left[\sum_{i=1}^{N}\left\langle\mathbf{r}_{i}^{2}(t)\right\rangle+\sum_{i=1}^{N}\left\langle\mathbf{r}_{i}^{2}(0)\right\rangle-2 \sum_{i=1}^{N}\left\langle\mathbf{r}_{i}(t) \mathbf{r}_{i}(0)\right\rangle\right]
$$

The so-called correlation factor, $F_{C}(t)$, is given by

$$
\begin{aligned}
F_{C}(t)= & 2 \sum_{i=1}^{N} \sum_{j>i}^{N}\left\langle\mathbf{r}_{i}(t) \mathbf{r}_{j}(t)\right\rangle+2 \sum_{i=1}^{N} \sum_{j>i}^{N}\left\langle\mathbf{r}_{i}(0) \mathbf{r}_{j}(0)\right\rangle \\
& -2 \sum_{i=1}^{N} \sum_{j>i}^{N}\left\langle\mathbf{r}_{i}(t) \mathbf{r}_{j}(0)\right\rangle .
\end{aligned}
$$

To perform each of the sums involved in the above equations, let us consider the system starting from a given initial configuration, where $p_{k}(m, s)$ is the probability of finding the first component of the $k$-mer $m$ in position $s$ at time $t=0$. Because the only interaction is the hard core exclusion, the final configuration $(t \rightarrow \infty)$ is completely at random. Therefore, the probability of finding the first component of the particle $m$ on the site $s$ in a chain composed of $L$ sites, such that $m \leqslant s \leqslant L$, is given by

$$
Q_{k}(m, s)=\frac{\left(\begin{array}{c}
s-1-(m-1)(k-1) \\
m-1
\end{array}\right)\left(\begin{array}{c}
L-s-k+1-(N-m)(k-1) \\
N-m
\end{array}\right)}{\left(\begin{array}{c}
L-N(k-1) \\
N
\end{array}\right)},
$$

where $N=\theta L / k$ is the number of $k$-mers in the chain $(\theta$ represents the coverage). The two factors in the numerator count the number of configurations of all particles to the left and right of particle $m$, respectively, and the denominator counts the total number of particle configurations on the chain.
In the same way, we can define the conditional probability to have the first component of the particle $n$ at site $r$, provided that the first component of the particle $m$ is at site $s$, as

$$
\begin{aligned}
& Q_{k}(n, r \mid m, s) \\
& =Q_{k}(m, s) \frac{\left(\begin{array}{c}
r-s-k-(n-m-1)(k-1) \\
n-m-1
\end{array}\right)\left(\begin{array}{c}
L-r-k+1-(N-m)(k-1) \\
N-m
\end{array}\right)}{\left(\begin{array}{c}
L-s-k+1-(N-m)(k-1) \\
N-m
\end{array}\right)} .
\end{aligned}
$$

With this formulation, we can write the sums defined in Eqs. (4) and (5) as

$$
\sum_{i=1}^{N}\left\langle\mathbf{r}_{i}^{2}(t)\right\rangle=\sum_{m=1}^{N} \sum_{s=k m-k+1}^{L-k(N-m+1)+1} s^{2} Q_{k}(m, s)
$$

and

$$
\sum_{i=1}^{N}\left\langle\mathbf{r}_{i}^{2}(0)\right\rangle=\sum_{m=1}^{N} \sum_{s=k m-k+1}^{L-k(N-m+1)+1} s^{2} P_{k}(m, s),
$$

where $P_{k}(m, s)$ coincides with $Q_{k}(m, s)$ for random initial distributions of particles. In the same way, we can write

$$
\begin{aligned}
\sum_{i=1}^{N} & \sum_{j>i}^{N}\left\langle\mathbf{r}_{i}(t) \mathbf{r}_{j}(t)\right\rangle \\
= & \sum_{m=1}^{N-1} \sum_{n=m+1}^{N} \sum_{s=k m-k+1}^{L-k(N-m+1)+1} \sum_{r=s+k+k(n-m-1)}^{L-k(N-m+1)+1} s Q_{k}(m, s) r \\
& \quad \times Q_{k}(n, r \mid m, s)
\end{aligned}
$$

and

$$
\begin{aligned}
\sum_{i=1}^{N} & \sum_{j>i}^{N}\left\langle\mathbf{r}_{i}(0) \mathbf{r}_{j}(0)\right\rangle \\
= & \sum_{m=1}^{N-1} \sum_{n=m+1}^{N} \sum_{s=k m-k+1}^{L-k(N-m+1)+1} \sum_{r=s+k+k(n-m-1)}^{L-k(N-m+1)+1} s P_{k}(m, s) r \\
& \times P_{k}(n, r \mid m, s) .
\end{aligned}
$$

Here $P_{k}(n, r \mid m, s)$ is the conditional probability to have the first component of the particle $n$ at site $r$, provided that the first component of the particle $m$ is at site $s$, at time $t=0$. This probability coincides with $Q_{k}(n, r \mid m, s)$ for random initial distributions of particles. Finally, we can write

$$
\begin{aligned}
& \sum_{i=1}^{N} \sum_{j>i}^{N}\left\langle\mathbf{r}_{i}(t) \mathbf{r}_{j}(0)\right\rangle \\
& \quad=\sum_{m=1}^{N} \sum_{n=1}^{N} \sum_{s=k m-k+1}^{L-k(N-m+1)+1} s^{2} Q_{k}(m, s) P_{k}(n, s) .
\end{aligned}
$$

These equivalences allow us to calculate analytically the MSD for both the tracer particle and the center of mass of the system, for any number of component of the $k$-mers, size of the box, and initial distribution of particles. For $k=1$ it is possible to recover the close expression obtained only for random initial distributions in Ref. [13]. 


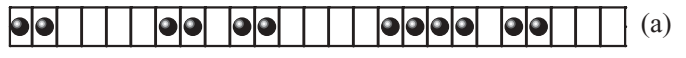

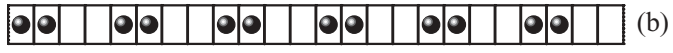
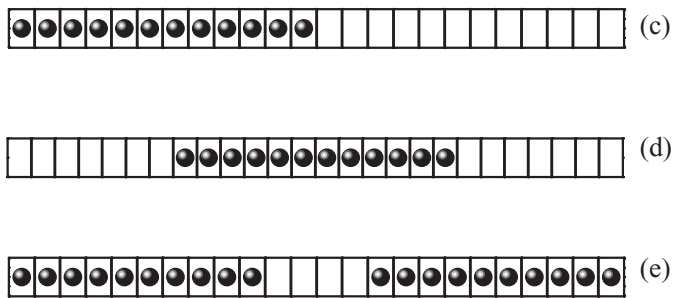

FIG. 1. Schematic representation of the initial distribution of dimers, $k=2$ : (a) random, (b) alternate, (c) one stack, (d) central stack, and (e) two identical stacks.

\section{RESULTS}

\section{A. The influence of the initial distribution}

In this section the influence of different initial configurations on the behavior of the MSD is analyzed. It is clear that the MSD for both the tracer and the center of mass of the system depends on four variables, $t, L, k$, and $\theta$, i.e., $S_{T}(t, L, k, \theta)$ and $S_{J}(t, L, k, \theta)$. Figure 1 shows a schematic representation of the initial distributions of particles used in the analysis: random [Fig. 1(a)]; alternate, where all particles are equidistant [Fig. 1(b); one stack [Fig 1(c); one central stack [Fig. 1(d)]; and two identical stacks [Fig. 1(e)]. The initial distribution of particles determines the behavior of the transient regime as well as the saturation. To verify this assumption, Fig. 2 shows the behavior of the MSD for the tracer particles [Fig. 2(a)] and for the center of mass of the system [Fig. 2(b)], for dimers $(k=2)$, in a box with $L=120$ and fixed concentration $\theta=0.1$. The same results are plotted in Fig. 3, for a coverage of $\theta=0.5$. The initial distributions used are described in the inset.

The Monte Carlo (MC) results are represented by symbols, while the solid lines are the values of the MSD in the asymptotic regime obtained by using the equations developed in the preceding section. These values are reported in the inset of each figure. It is observed that the initial configuration substantially affects the behavior of the MSD, both in the transient regime and in the saturation.

Before discussing the results, let us mention some details of the MC simulation. To mimic a box, a one-dimensional finite chain of length $L$ with reflecting borders is considered. There are $N=\theta L / k$ diffusing particles of size $k$ that can jump to the right or to the left with equal transition probabilities per unit time $J_{0}=1 / 2$. No interaction, except a hard core, is considered (the basic assumption of the SFD problem). In one MC step there are $2 \mathrm{~N}$ possible processes, and one of these processes is chosen with the corresponding probability. The classical fixed time step MC and kinetic MC methods [28] are used to obtain the simulation results. Because the nonexternal field is considered, the results obtained by using both methods are in agreement, according to the study developed in Ref. [29].

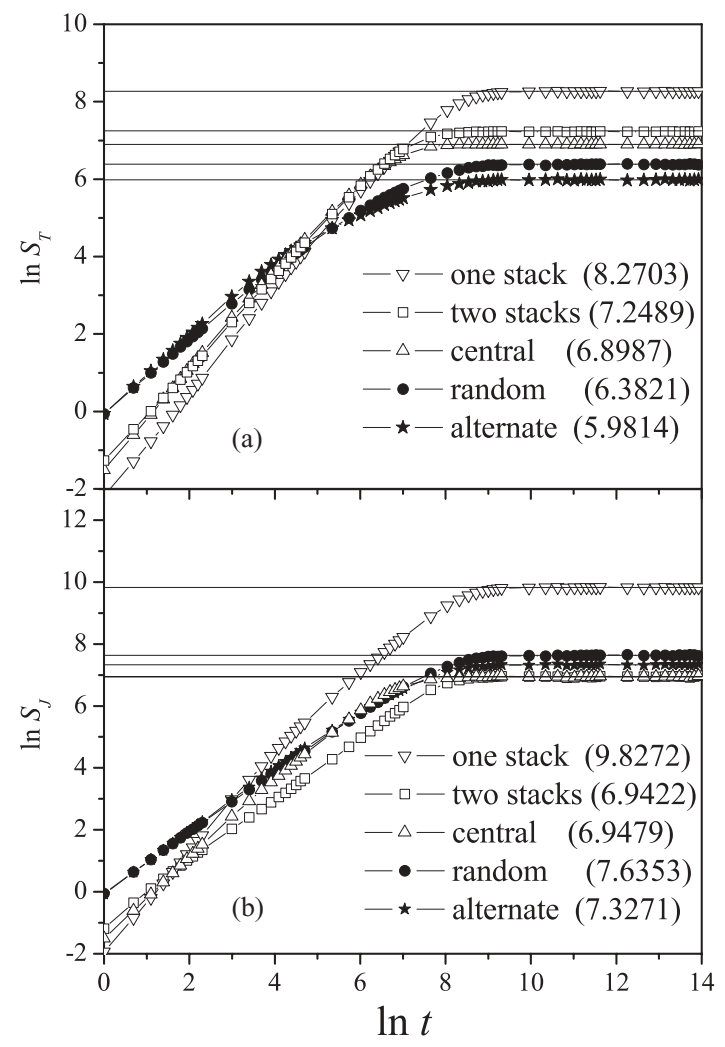

FIG. 2. MSD for dimers in a box of size $L=120$ and concentration $\theta=0.1$ : (a) tracer particles and (b) center of mass of the system.

It is well discussed in the literature that the diffusion of a tagged particle in a box presents a normal diffusion regime up to a characteristic time beyond which different authors claim that the dynamic of the system is dominated by particle collisions, leading to single-file behavior. In Fig. 2(a), one can observe this behavior for random and alternate initial distributions. The slope of the $S_{T}$ in the subdiffusive regime is $\alpha \approx 0.5$ for the random initial distribution, while for the alternate initial distribution it is slightly smaller $(\alpha \approx 0.44)$. For higher coverage [Fig. 3(a)], normal diffusion is not observed for these two cases.

However, for an initial configuration where the particles are completely stacked, the results show that in all cases the slope of the transient regime is $\alpha>1$, confirming that the particles will be forced to move, on average, in one direction more than in the other, like in a biased diffusion. This characteristic is observed for both coverages. Even when in the transient regime, the stacked particles present similar superdiffusive behavior and the corresponding saturations are different.

For $S_{J}$, the slope of the transient regime, for random and alternate initial distributions, is $\alpha=1$ and does not depend on coverage [see Figs. 2(b) and 3(b)], while for stacked initial distributions the slope depends on the coverage.

For $\theta=0.1$ [Fig. 2(b)], the slope for the two-stack initial distribution is $\alpha \approx 1$, while for the one-stack initial distribution the slope changes from $\alpha \approx 1.60$ to $\alpha \approx 1.2$. For the central stack initial distribution, the slope changes from $\alpha \approx 1.28$ to $\alpha \approx 1$. 


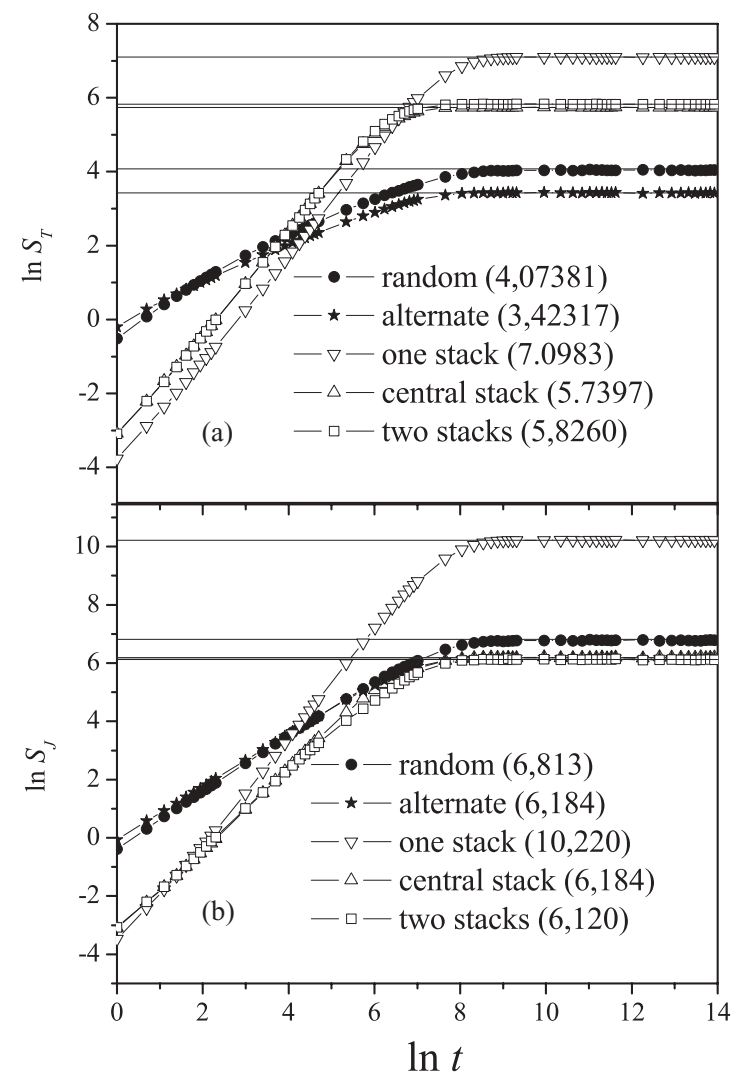

FIG. 3. MSD for dimers in a box of size $L=120$ and concentration $\theta=0.5$ : (a) tracer particles and (b) center of mass of the system.

For $\theta=0.5$ [Fig. 3(b)], the slope for central and two-stack initial distributions is $\alpha \approx 1.44$, while for one stack it changes from $\alpha \approx 1.44$ to $\alpha \approx 1.9$.

In Figs. 4(a) and 4(b) the saturation values of $S_{T}$ and $S_{J}$ are plotted as a function of coverage, for $k=2, L=120$, and different initial configurations of particles. The analytical results are in perfect agreement with the MC simulation. Note that, in the alternate case, the coverage depends on the ratio between $k$ and $L$. Moreover, with this condition, the maximum coverage is given by $\theta_{\max }=\frac{k}{k+1}$.

Although the results for both $S_{T}^{\text {sat }}(L, k, \theta)$ and $S_{J}^{\text {sat }}(L, k, \theta)$ at saturation, obtained through the combinatorial treatment, are numerically exact, it would be desirable to have a manageable expression for both coefficients.

In Ref. [13], the authors have obtained an approximate expression for $S_{T}$, for $k=1$ (monomers). This is valid only for random initial distributions of particles.

Following the same methodology, we have proposed the following expressions for the MSD in the case of random initial distribution:

$$
S_{T}^{\mathrm{sat}}(L, k, \theta) \approx \frac{k}{3} \frac{(1-\theta)}{\theta}\left[1-\left(\frac{k-1}{k}\right) \theta\right] L,
$$

for the tracer particles, and

$$
S_{J}^{\mathrm{sat}}(L, k, \theta) \approx \frac{k}{6}(1-\theta)\left[1-\left(\frac{k-1}{k}\right) \theta\right] L^{2},
$$

for the center of mass.

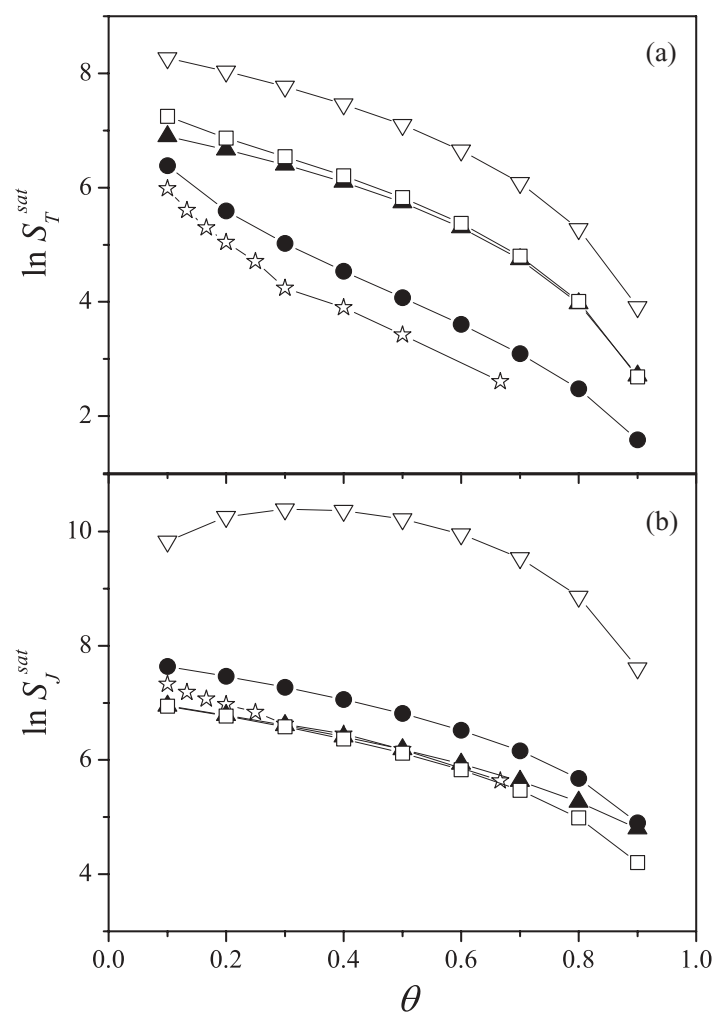

FIG. 4. Analytical results for the MSD at saturation for dimers in a box of size $L=120$, as a function of the concentration: (a) tracer particles and (b) center of mass of the system. Symbols are as follows: solid circles, random; empty stars, alternate; empty triangles, one stack; solid triangles, central stack; empty squares, two stacks.

As can be observed, see Figs. 5(a) and 5(b), the agreement between the combinatorial analytical solutions and the proposed functions is excellent for a large range of the parameters $k$ and $L$.

For the other initial distributions, like the alternate distribution, the MSD for tracer particles can be approximated by the function given in Eq. (13), divided by 3. While, for the stacked initial distributions, the MSD at saturation, for the tracer particles, is almost independent of the size of the $k$-mers, depending on the square of the vacancy and the length of the chain, $S_{T}^{\text {sat }}(L, k, \theta) \approx C(1-\theta)^{2} L^{2}$, where $C$ depends on the initial distribution ( $C=\frac{1}{3}$ for one stack, $C=\frac{1}{12}$ for one central stack, and $C=\frac{\sqrt{2 \pi}}{24}$ for two stacks.). However, the MSD, corresponding to the center of mass $S_{J}^{\mathrm{sat}}(L, k, \theta)$, cannot fit adequately in any other case.

Next, the effect of the initial distribution on the transient regime is discussed. One of the most controversial aspects of the SFD, is the crossover between normal and subdiffusive behaviors. The results show that, the diffusion of a tagged particle presents a normal diffusion regime, i.e., the MSD is proportional to $t: S_{T}(t)=2 D t$, where $D$ is the diffusion coefficient. This relation is valid up to a characteristic time, beyond which, on average, particles start colliding with their neighbors. Then, as a consequence of the interaction between particles and the one-dimensional character of the problem, the tagged particle shows a subdiffusive behavior of the form $S_{T}(t)=2 F_{H} t^{1 / 2}$, 


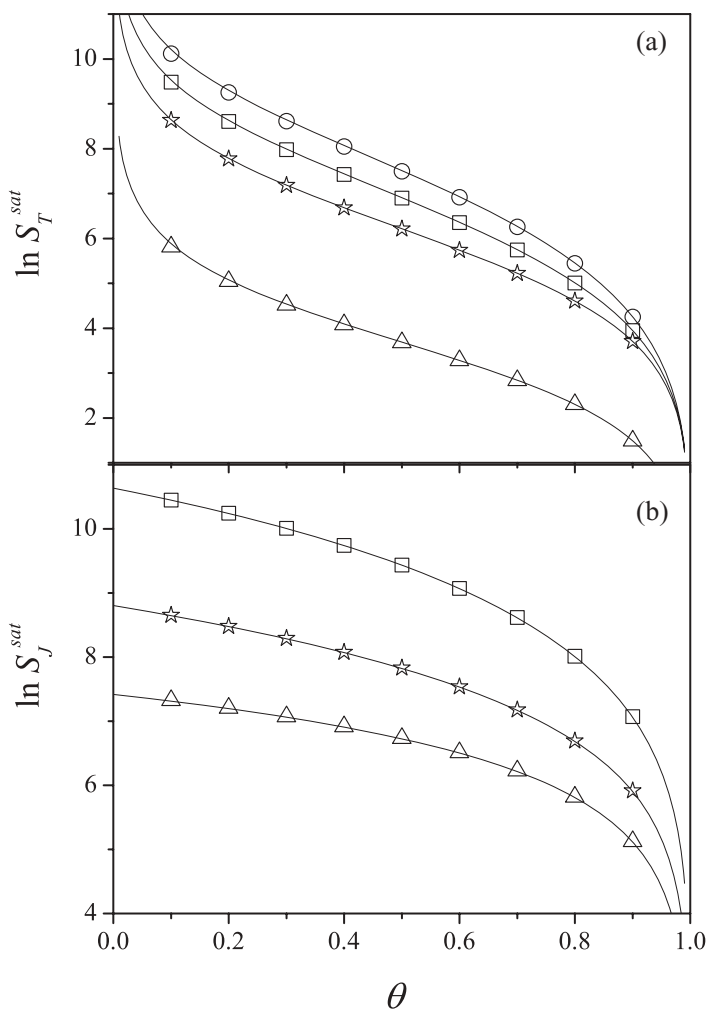

FIG. 5. Comparison between analytical results and approximate functions for the MSD at saturation, with random initial configurations. (a) MSD of the tracer particles, $S_{T}^{\text {sat }}(k, L, \theta)$ : circles, $k=10$ and $L=1000$; squares, $k=5$ and $L=1000$; stars, $k=2$ and $L=1000$; and triangles, $k=1$ and $L=1000$. (b) MSD of the center of mass of the particles, $S_{J}^{\text {sat }}(k, L, \theta)$ : squares, $k=5$ and $L=500$; stars, $k=2$ and $L=200$; and triangles, $k=1$ and $L=100$.

where $F_{H}$ is a characteristic coefficient. This subdiffusive regime lasts forever in infinite systems, but it crosses over to saturation or normal diffusion in finite systems, depending on the applied boundary conditions [13]. It is assumed that the subdiffusive regime of a single tagged particle is the signature of the interacting system. On the contrary, the diffusion of the center of mass is always normal; i.e., it increases linearly with time, without showing any signature of the interacting system. This behavior has been observed when the initial configuration of particles is random or, as has been shown in Ref. [21], for a particular arrangement where particles are equally spaced.

In order to discuss this assumption, Fig. 6 shows the number of collisions as a function of time in a log-log plot for three different initial conditions. As can be observed, for the one-stack initial condition the number of collisions is dominant at the beginning and decreases to reach a constant value. This behavior is also observed for the other two-stack initial conditions. On the other hand, the number of collisions for the alternate initial distribution is almost zero at the beginning, reaching the same constant value. However, for random initial distributions, the number of collisions does not change in time, having the same constant value which is directly proportional to the coverage. Surprisingly, from the simple inspection of these curves, one can conclude that the change in the slope of the MSD for random and alternate initial distributions cannot

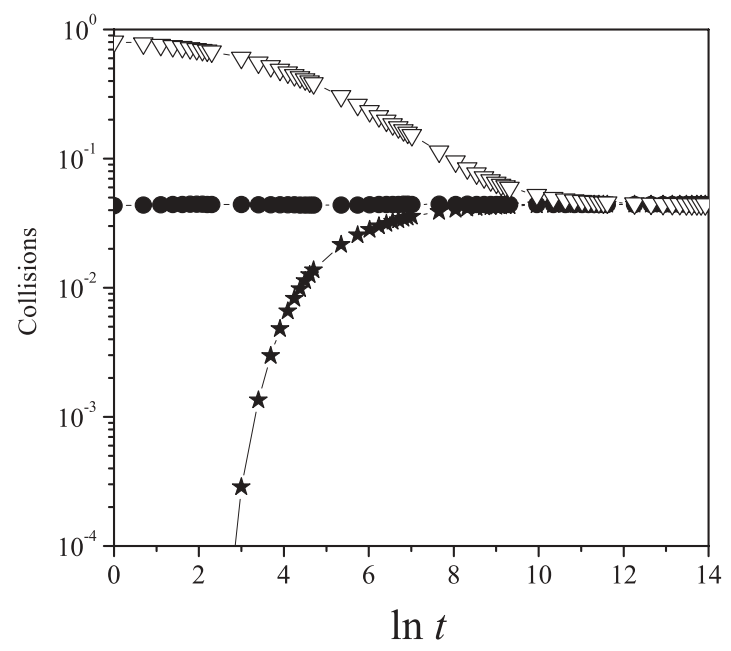

FIG. 6. Number of collisions as a function of time, for dimers $k=2$, in a box of $L=120$ at coverage $\theta=0.1$. Three different initial distributions of particles are used: solid circles, random; solid stars, alternate; empty triangles, one stack.

be attributed to the number of collisions. Particularly, because the random initial distribution presents the same number of collisions at all times.

To attain a better understanding of the origin of the crossover between normal and subdiffusive regimes, Figure 7 shows the first two terms and the third term of Eq. (4) versus the logarithm of time, for three different initial distributions. The term $\frac{1}{N}\left[\sum_{i=1}^{N}\left\langle\mathbf{r}_{i}^{2}(t)\right\rangle+\left\langle\mathbf{r}_{i}^{2}(0)\right\rangle\right]$ represents the position of the particle at time $t$ added to the position of the particle at the initial time. These terms are independent of each other. However, the term $2 \sum_{i=1}^{N}\left\langle\mathbf{r}_{i}(t) \mathbf{r}_{i}(0)\right\rangle$ represents the autocorrelation between the position of the particle at time $t$ with the position of the particle at time $t=0$. The competition between these two terms gives rise to the temporal dependence of the MSD. In fact, at the beginning the two terms are nearly equal. Then, the first term starts being greater than the second. At that time, we must distinguish two behaviors: (a) for random and alternate initial distributions, the diffusion goes from normal to anomalous (subdiffusion); and (b) for stacked initial distributions, the exponent changes from $\alpha \approx 1.44$ to $\alpha \approx 1.09$. Finally, in all cases, the MSD reaches a constant value.

Following the latter arguments, one can observe that the final stage for the MSD of the tracer particles with a one-stack initial arrangement is the largest one, while that of a tracer starting, say, from an alternate configuration is the smallest. The random initial distribution is in between. That cannot be explained by the number of collisions, given that with time the number of collisions saturates to the same value. In fact, the behavior of the MSD for the tracer is not determined by the collisions between particles, but is due to the balance in the autocorrelations in Eq. (4) (Similar arguments can be used for the MSD of the center of mass of the system). In Fig. 7, one can observe that the biggest difference between both terms is for the one-stack initial configuration, while the smallest corresponds to the alternate initial configuration. In between, is the random distribution. Therefore, both the transient regime and the saturation are explained by the balance between the 


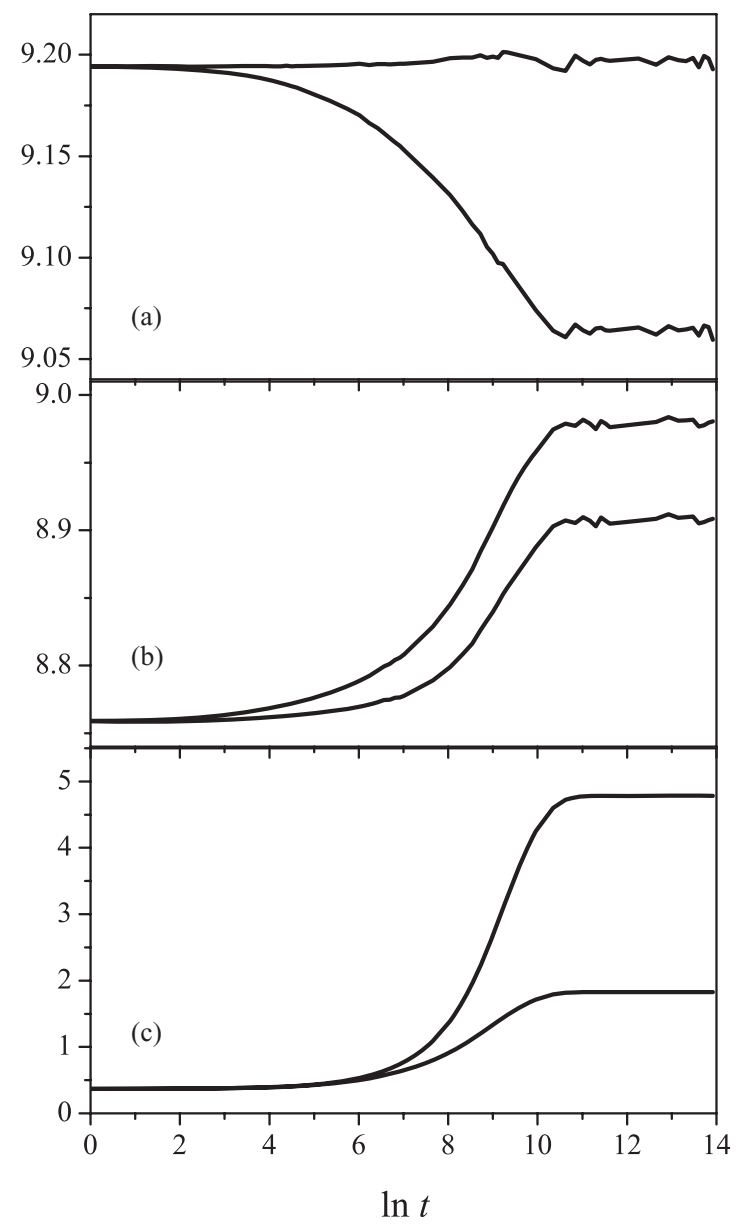

FIG. 7. Comparison between the first two terms (upper curve) and the third term (lower curve) in Eq. (4) for $k=2, L=240$, and $\theta=$ 0.2. (a) Random initial distribution; (b) alternate initial distribution; (c) stacked initial distribution. In the vertical axis, the number of steps is in lattice units divided by $10^{5}$.

terms in Eq. (4) and not by the collisions between particles, as argued by many authors.

\section{B. Transient regime for random initial distribution}

To attain a deeper understanding of the behavior of the MSD in the transient regime, in what follows is considered the dependence of the MSD with the size of the box $L$, the coverage $\theta$, and the size of the particle $k$, for the random distribution. The particles of size $k$ are on average a distance $\langle\ell\rangle$ apart (the distance is measured from the first component of the $k$-mer), and then the size of the system is $L=\langle\ell\rangle N$ and the coverage can be written as $\theta=k /\langle\ell\rangle$. Thus, the original lattice can be rescaled in such a way that the equivalent coverage is proportional to the inverse of the new average distance between particles, $\left\langle\ell^{\prime}\right\rangle$; therefore

$$
\theta_{\mathrm{eq}}=\frac{1}{\left\langle\ell^{\prime}\right\rangle}=\frac{1}{\ell-(k-1)}=\frac{\theta}{k-(k-1) \theta} .
$$

By replacing the expression of equivalent coverage in Eq. (2.21a) of Ref. [13] and considering the limit of the large size system, $L \rightarrow \infty$, the expression for the short-time

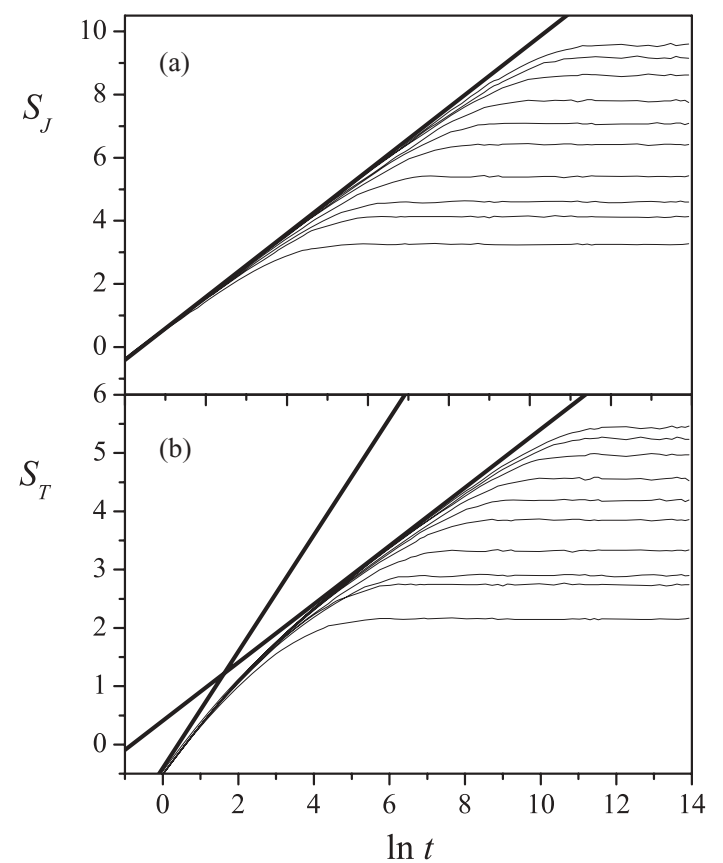

FIG. 8. The behavior of the MSD as a function of time and $L$ for $k=2$ at $\theta=0.5$ for (a) the center of mass of the system and (b) the tracer particles. The slope of the straight lines correspond to the expression obtained in Eqs. (16) and (17), respectively. The Monte Carlo simulations correspond to different sizes of the box ( $L=20,30,40,60,100,140,200,300,400$, and 500, from bottom to top).

behavior of $S_{T}$ can be obtained by

$$
S_{T}(k, \infty, \theta, t)=2 \frac{k(1-\theta)}{[k-(k-1) \theta]} a^{2} J_{0} t,
$$

where $J_{0}=1 / 2$ is the probability to jump to the right or to the left and $a$ is the lattice constant. Proceeding in the same way with Eq. (2.21b) of Ref. [13], the intermediate-time behavior of $S_{T}$ and be obtained by

$$
S_{T}(k, \infty, \theta, t)=\frac{2 k(1-\theta)}{\theta} a^{2} \sqrt{\frac{J_{0} t}{\pi}} .
$$

In Ref. [27], the authors have obtained the exact calculation of the jump diffusion coefficient $D_{J}$ for noninteracting $k$-mers in a one-dimensional infinite chain. Using the expression for $D_{J}$, the time dependence of $S_{J}$ in the transient regime can be easily obtained. The expression of $S_{J}$ is the same as that of the short-time behavior $S_{T}$. Therefore, the first stage of the MSD for tracer particles coincides with the MSD corresponding to the center of mass of the system.

Figure 8 shows the behavior of the MSD as a function of time and $L$ for $k=2$ at half coverage, $\theta=0.5$, for a random initial distribution. The slope of the straight lines correspond to the expressions obtained in Eqs. (16) and (17) and they are the asymptotic limits of the MSD.

Figure 9 shows the dependence of the MSD for different size of the particles and different coverages. It can be observed that the agreement between the analytical results and the simulations is rather good, despite that the size of the systems is not so large. 


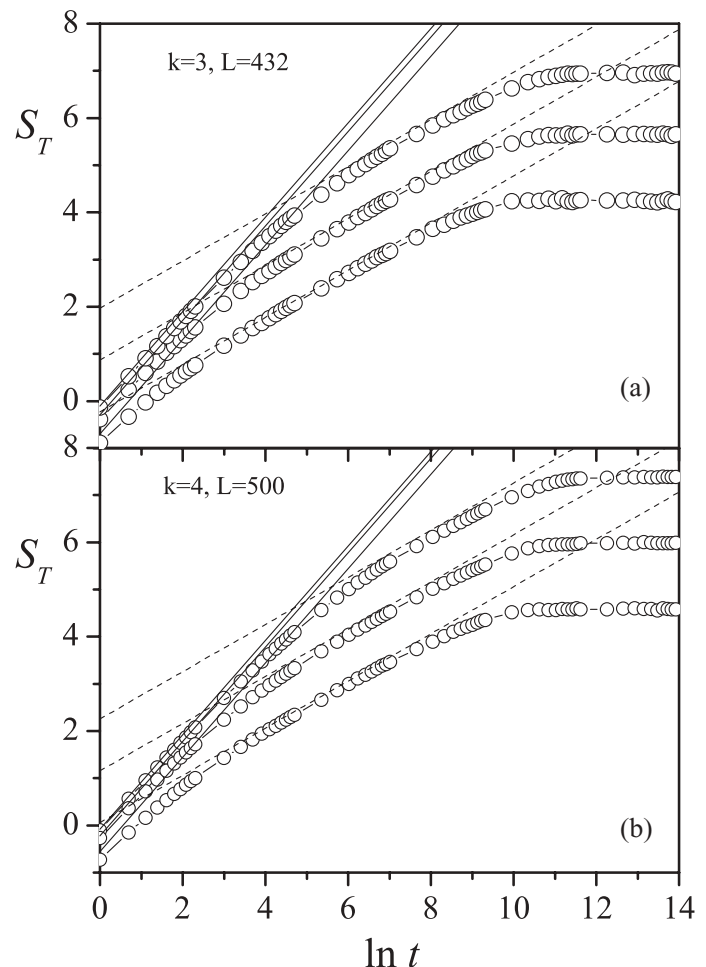

FIG. 9. The behavior of the MSD for tracer particles as a function of time for different coverages and sizes of the particles: (a) $k=3$ and $L=432$; (b) $k=4$ and $L=500$. In both cases, the coverages are $\theta=0.25,0.50$, and 0.75 , from top to bottom. The solid lines correspond to Eq. (16) while the dashed lines correspond to Eq. (17).

\section{CONCLUSIONS}

In this work, the influence of the initial distribution in SFD of particles confined in a box is studied. An analytical solution for the MSD at saturation, for both the tracer and the center of mass of $k$-mers (particles with multiple-site occupancy) is obtained by means of combinatorial technics. The analytical solution is in agreement with the MC simulation. Random, alternate, and stacked initial distributions are used. It is observed that the influence of the initial distribution affects not only the saturation regime but also the transient regime. In fact, for the alternate and random initial distributions, the classical crossover from normal to subdiffusive regimes, in the MSD corresponding to the tracer particles, is observed for any size of particles and boxes. It is more clear and marked for the alternate initial distribution than for the random initial distribution. The MSD corresponding to the center of mass presents a normal diffusive behavior. However, for stacked initial distributions, the transient regime is slightly more complicated. In fact, a superdiffusive regime is observed for all the analyzed cases. The slope of the MSD for tracer particles is greater than 1. The MSD corresponding to the center of mass presents a superdiffusive regime too. The slope is also greater than 1 and depends on the coverage. This behavior is easy to understand; as soon as the particles start to move, they have a higher probability to jump in one direction than in the other because of the occupancy. This behavior is similar to a biased diffusion. Then, the origin of different diffusion regimes is studied. They are not originated by the collisions between particles, but because the behavior of the autocorrelation of the position at time $t$ with the position at time $t=0$. Both the transient regime and the saturation are explained by the balance between those terms. Particularly, the difference between the saturation values for different initial configurations for both MSDs cannot be explained by the number of collisions, given that with time the number of collisions saturates to the same value. Finally, analytical approximations for the MSD for both tracer particles and the center of mass are obtained, provided that the initial distribution of particles is random. This solution is obtained by means of simple rescaling arguments.

\section{ACKNOWLEDGMENTS}

The authors thank Dr. P. Centres and Dr. O. Furlong for discussions. This work is partially supported by the CONICET (Argentina).
[1] A. L. Hodgkin and R. D. Keynes, J. Physiol. 128, 61 (1955).

[2] R. J. Ellis and A. P. Milton, Nature (London) 425, 27 (2003).

[3] B. Lin, M. Meron, B. Cui, S. A. Rice, and H. Diamant, Phys. Rev. Lett. 94, 216001 (2005).

[4] O. G. Berg, R. B. Winter, and P. H. von Hippel, Biochemistry 20, 6929 (1981).

[5] S. E. Halford and J. F. Marko, Nucl. Acids Res. 32, 3040 (2004).

[6] M. A. Lomholt, T. Ambjörnsson, and R. Metzler, Phys. Rev. Lett. 95, 260603 (2005).

[7] M. A. Lomholt, T. Ambjörnsson, and R. Metzler, Phys. Rev. E 82, 031201 (2010).

[8] C. Dekker, Nat. Nanotechnol. 2, 209 (2007).

[9] T. E. Harris, J. Appl. Prob. 2, 323 (1965).

[10] P. M. Richards, Phys. Rev. B 16, 1393 (1977).

[11] P. A. Fedders, Phys. Rev. B 17, 40 (1978).
[12] S. Alexander and P. Pincus, Phys. Rev. B 18, 2011 (1978).

[13] H. van Beijeren, K. W. Kehr, and R. Kutner, Phys. Rev. B 28, 5711 (1983).

[14] A. Arratia, Ann. Probab. 11, 362 (1983).

[15] C. Rödenbeck, J. Kärger, and K. Hahn, Phys. Rev. E 57, 4382 (1998).

[16] C. L. Aslangul, Europhys. Lett. 44, 284 (1998).

[17] M. Kollmann, Phys. Rev. Lett. 90, 180602 (2003).

[18] A. Brzank and G. M. Schütz, J. Stat. Mech.: Theory Exp. (2007) P08028; arXiv:cond-mat/0611702.

[19] L. Lizana and T. Ambjörnsson, Phys. Rev. Lett. 100, 200601 (2008).

[20] T. Ambjörnsson, L. Lizana, M. A. Lomholt, and R. J. Silbey, J. Chem. Phys. 129, 185106 (2008).

[21] P. M. Centres and S. Bustingorry, Phys. Rev. E 81, 061101 (2010). 
[22] V. Pereyra, A. Milchev, and V. Fleurov, Phys. Rev. E 50, 4636 (1994); G. Zgrablich, V. Pereyra, M. Ponzi, and J. Marchese, AIChE J. 32, 1158 (1986).

[23] J. W. Haus and K. Kehr, Phys. Rep. 150, 263 (1987).

[24] S. Havlin and D. Ben-Avraham, Adv. Phys. 36, 695 (1987).

[25] J.-B. Delfau, C. Coste, and M. Saint Jean, Phys. Rev. E 84, 011101 (2011).
[26] D. Lucena, D. V. Tkachenko, K. Nelissen, V. R. Misko, W. P. Ferreira, G. A. Farias, and F. M. Peeters, Phys. Rev. E 85, 031147 (2012).

[27] A. J. Ramirez-Pastor, T. P. Eggarter, V. D. Pereyra, and J. L. Riccardo, Phys. Rev. B 59, 11027 (1999).

[28] F. M. Bulnes, V. D. Pereyra, and J. L. Riccardo, Phys. Rev. E 58, 86 (1998).

[29] V. Ruiz Barlett, J. J. Bigeon, M. Hoyuelos, and H. O. Martin, J. Comp. Phys. 228, 5740 (2009). 